\title{
A Sex Allocation Theory for Vertebrates: Combining Local Resource Competition and Condition- Dependent Allocation
}

\author{
Geoff Wild $^{1, *}$ and Stuart A. West ${ }^{2, \dagger}$
}

1. Department of Applied Mathematics, University of Western Ontario, London, Ontario N6A 5B7, Canada;

2. Institute of Evolutionary Biology, School of Biological Sciences, University of Edinburgh, Edinburgh EH9 3JT, United Kingdom

Submitted March 12, 2007; Accepted July 13, 2007;

Electronically published September 18, 2007

ABSTRACT: Tests of sex allocation theory in vertebrates are usually based on verbal arguments. However, the operation of multiple selective forces can complicate verbal arguments, possibly making them misleading. We construct an inclusive fitness model for the evolution of condition-dependent brood sex ratio adjustment in response to two leading explanations for sex ratio evolution in vertebrates: the effect of maternal quality on the fitness of male and female offspring (the Trivers-Willard hypothesis [TWH]) and local resource competition (LRC) between females. We show (1) the population sex ratio can be either unbiased or biased in either direction (toward either males or females); (2) brood sex ratio adjustment can be biased in either direction, with high-quality females biasing reproductive investment toward production of sons (as predicted by the TWH) or production of daughters (opposite to predictions of the TWH); and (3) selection can favor gradual sex ratio adjustment, with both sons and daughters being produced by both high- and low-quality mothers. Despite these complications, clear a priori predictions can be made for how the population sex ratio and the conditional sex ratio adjustment of broods should vary across populations or species, and within populations, across individuals of different quality.

Keywords: kin selection, local resource competition, mathematical model, sex allocation, sex ratio, Trivers-Willard effect.

\footnotetext{
* E-mail: gwild@uwo.ca.

† E-mail: stu.west@ed.ac.uk.
}

Am. Nat. 2007. Vol. 170, pp. E112-E128. (C) 2007 by The University of Chicago. 0003-0147/2007/17005-42458\$15.00. All rights reserved.

DOI: $10.1086 / 522057$
Sex allocation represents one of the most productive and successful areas of evolutionary biology. Theory predicts a number of situations in which individuals are expected to adjust their allocation to male and female reproduction, and there is a large empirical literature supporting these predictions (Charnov 1982; Frank 1998; Hardy 2002). Much of the clearest support for sex allocation theory comes from studies of invertebrates (West and Sheldon 2002; West et al. 2005). A key reason for this success has been the interplay between theoretical and empirical research, with the development of specific models that can be tested with added precision in specific systems (e.g., Trivers and Hare 1976; Werren 1980; Charnov et al. 1981; Charnov 1982; Herre 1985; Yamaguchi 1985; Boomsma and Grafen 1991; Taylor and Crespi 1994; Shuker et al. 2005).

In contrast to data from invertebrates, those from vertebrates such as birds and mammals have provided equivocal support for sex allocation theory (Brown and Silk 2002; Cockburn et al. 2002; West and Sheldon 2002; Schino 2004; Sheldon and West 2004). A major problem for the application of sex allocation theory to vertebrates such as birds and mammals is the lack of interplay between theoretical and empirical research. This can be illustrated by considering the most famous sex allocation prediction for vertebrates, the Trivers and Willard (1973) hypothesis (TWH). Trivers and Willard (1973) envisaged a mammal population in which (1) females in better condition have more resources for reproduction and hence produce higher-quality offspring and (2) intense competition for mates between males leads to male fitness increasing much more rapidly with quality than female fitness. In this case, theory predicts that poor-quality mothers should produce daughters and high-quality mothers should produce sons. This model has been applied to a huge range of vertebrates, especially ungulates, primates, marsupials, and birds (Brown and Silk 2002; Cockburn et al. 2002; Schino 2004; Sheldon and West 2004).

Empirical tests of the Trivers and Willard (1973) hy- 
pothesis in vertebrates are usually based on verbal arguments, which can lead to three problems. First, some studies have focused on whether overall population sex ratios are biased (reviewed by West and Sheldon [2002]). In general, this approach lacks a clear theoretical basis, because whether the overall population sex ratio should even be biased, let alone in what direction, can depend on a number of life-history details that can be difficult to measure (Frank 1987, 1990; Pen and Weissing 2000a, 2002). Second, multiple factors can influence sex allocation in vertebrates, which complicates matters. For example, in many species in which the Trivers-Willard effect could be expected, limited dispersal by females can lead to local resource competition (LRC) between related females, which reduces the marginal fitness gain of producing daughters (Clark 1978; Taylor 1981; Clutton-Brock et al. 1982; Silk 1983). When multiple factors act, then verbal arguments can give misleading predictions (Frank 1990). Third, the possibility of asymmetrical transmission of quality from parent to offspring can lead to very different predictions. For example, if only daughters inherit aspects of quality from mothers, such as maternal rank, then this can select for high-quality mothers to be more likely to produce daughters - the opposite direction to that predicted by Trivers and Willard (Leimar 1996).

Here we address these problems by modeling the effects of when both LRC and the Trivers-Willard effect can occur simultaneously in the same species. Our aim is to determine whether clear predictions can be made for either the overall population sex ratio or the direction of brood sex ratio adjustment across individuals of different quality within a population. We distinguish between absolute predictions for a given population or species (e.g., the offspring sex ratio should be male biased) and relative predictions across populations or species (e.g., the offspring sex ratio should be more male biased in species or populations in which there is more female philopatry). For clarity, we model the simplest possible scenario: the Trivers-Willard effect is allowed for by having offspring quality determined by patch quality and two types of patch quality-high and low; LRC is allowed for between females by having only a fraction $d$ of females disperse from their natal patch and complete male dispersal. In the discussion, we relate our predictions to existing empirical data.

\section{The Model}

\section{Preliminaries}

We consider a diploid sexual species undergoing discrete, nonoverlapping generations. We suppose that the population is divided into an infinite number of breeding patches, with each breeding patch supporting exactly one fertilized female.

Breeding patches themselves are assumed to vary in quality. Specifically, we assume that there exist two patch "types" (indexed; $i=1,2$ ). One patch type (type 1) is of high quality, while the other type (type 2 ) is of low quality. We let $p_{i}$ denote the frequency of a type $i$ patch, and we suppose that these frequencies remain constant over time. A key assumption here is that patch quality and maternal quality are inextricably linked: a mother is of high (low) quality because she breeds on a patch of high (low) quality.

A parent is assumed to make some fixed investment in offspring, and in turn this investment influences offspring survival. A parent can manipulate the sex ratio of its brood, then, by altering the proportion of the fixed investment it gives to sons. We refer to this proportion as parental "sex allocation behavior" and denote its population average as $z_{i}$. We attach the subscript $i$ to $z$ to remind us that sex allocation behavior may be expressed differently depending on the quality of the parent's breeding patch. It is the joint evolution of $z_{1}$ and $z_{2}$ that is the focus of this article.

We will treat $z_{1}$ and $z_{2}$ as behaviors expressed by a mother. This assumption is made only because it improves the clarity of the exposition. Our results are not affected by treating $z_{1}$ and $z_{2}$ as behaviors expressed by a father, but that is not the case in more general models (e.g., Pen and Weissing 2002; Wild and Taylor 2005).

\section{Life Cycle}

Each generation consists of a series of events. These are outlined below in their order of occurrence (see table 1 for a list of symbols and definitions).

1. Birth. A fertilized female gives birth to $K$ sons and $K$ daughters, where $K$ is very large (ideally infinite) and independent of patch quality. Note that brood sex ratios at this stage are unbiased.

2. Maternal Investment. Mothers express conditional sex allocation behaviors. At the end of this stage, the brood on a type $i$ patch consists of $K z_{i}$ sons and $K\left(1-z_{i}\right)$ daughters. Note that thinking of $z_{i}$ as a probability of survival is merely a technical device used to guide our modeling efforts. The ratio $\left(1-z_{i}\right) / z_{i}$ could also be interpreted as the "birth sex ratio" of the brood produced by a type $i$ mother.

3. Dispersal. Sex-specific dispersal patterns have been reported in many species of vertebrates (Greenwood 1980). To reflect common dispersal patterns in mammals, we assume complete dispersal of males but only partial dis- 
Table 1: Explanation of the principal notation used in the main text

\begin{tabular}{ll}
\hline Symbol & Explanation \\
\hline$d$ & Rate of natal dispersal by females \\
$\delta_{\mathrm{m}}, \delta_{\mathrm{f}}$ & Deviant increase in the level of investment in sons and daughters, respectively $\left(\delta_{\mathrm{f}}=-\delta_{\mathrm{m}}\right)$ \\
$K$ & Size of brood at the end of the maternal investment period (stage 2 of life cycle) \\
$N_{\mathrm{f}, i}$ & Effective number of females competing on a type $i$ patch \\
$N_{\mathrm{m}}$ & Effective number of males competing on any patch \\
$p_{i}$ & Frequency of type $i$ patches $\left(p_{1}=1-p_{2}\right)$ \\
$r_{i}$ & Strength of local resource competition on a type $i$ patch (a number between 0 and 1$)$ \\
$v_{\mathrm{f}, i}, v_{\mathrm{m}, i}$ & Reproductive value of female and male, respectively, born on a type $i$ patch, measured at birth \\
$v_{\mathrm{f},(i, j)}, v_{\mathrm{m},(i, j)}^{\prime}$ & Reproductive value of female and male, respectively, born on a type $i$ patch but competing on a \\
$w_{\mathrm{f}, i}, w_{\mathrm{m}, i}$ & type $j$ patch \\
$\Delta W_{\mathrm{f}, i}, \Delta W_{\mathrm{m}, i}$ & Intrasexual competitive abilities of a female and male, respectively, born on a type $i$ patch \\
$\Delta W_{i}=\Delta W_{\mathrm{m}, i}+\Delta W_{\mathrm{f}, i}$ & Inclusive fitness effect through daughters and sons, respectively, born on a type $i$ patch \\
$z_{i}$ & Overall inclusive fitness effect of the production of sons on a type $i$ patch (recall, $\left.\delta_{\mathrm{f}}=-\delta_{\mathrm{m}}\right)$ \\
\hline & Proportion of reproductive investment the average type $i$ mother allocates to the production of sons \\
\hline
\end{tabular}

persal by females (let $d$ denote the female dispersal rate). For simplicity, we do not impose a survival cost on dispersing individuals.

In addition to the assumptions outlined above, we assume that dispersal rates do not evolve and do not depend on habitat quality. The consequences of relaxing these assumptions are not trivial and have been explored elsewhere (Leturque and Rousset 2003, 2004; Wild and Taylor 2004; Wild et al. 2006).

4. Male-Male Competition. Each female is fertilized by exactly one male; however, the mating success of a male is variable. Males born on a high-quality patch enjoy a competitive advantage over males born on a low-quality patch. If $w_{\mathrm{m}, i}$ denotes the competitive ability of a male born on a type $i$ patch, then the effective amount of malemale competition for access to any given female is simply

$$
N_{\mathrm{m}}=K \sum_{j} w_{\mathrm{m}, j} p_{j} z_{j}
$$

By the assumption $w_{\mathrm{m}, 1}>w_{\mathrm{m}, 2}$, the relative competitive advantage enjoyed by the son of a type 1 mother is $w_{\mathrm{m}, 1} / w_{\mathrm{m}, 2}>1$.

5. Female-Female Competition. Fertilized females from stage 4 of the life cycle compete for access to the vacated breeding site. As before, we posit a competitive advantage for females born on a high-quality patch. On a type $i$ patch the effective number of competing females is

$$
N_{\mathrm{f}, i}=K\left[(1-d) w_{\mathrm{f}, i}\left(1-z_{i}\right)+d \sum_{j} w_{\mathrm{f}, j} p_{j}\left(1-z_{j}\right)\right],
$$

where $w_{\mathrm{f}, i}$ is the competitive ability of a female born on a type $i$ patch. We assume that daughters born to type 1 mothers usually enjoy a relative competitive advantage over those born to type 2 mothers. That is, $w_{\mathrm{f}, 1} / w_{\mathrm{f}, 2} \geq 1$.

Note that when $w_{\mathrm{m}, 1} / w_{\mathrm{m}, 2}=w_{\mathrm{f}, 1} / w_{\mathrm{f}, 2}$, the model describes a situation in which parents breeding on a highquality patch enjoy only a fecundity advantage over those breeding on a low-quality patch. Leturque and Rousset (2003) have investigated the consequences of fecundity advantage. In this article, we suppose that superior patch quality has sex-specific consequences for offspring competitive ability. Specifically, we assume

$$
\frac{w_{\mathrm{m}, 1}}{w_{\mathrm{m}, 2}}>\frac{w_{\mathrm{f}, 1}}{w_{\mathrm{f}, 2}} \geq 1
$$

To be clear, inequality (1) means that the investments made in sons by mothers in good condition (i.e., mothers breeding on a high-quality patch) are always rewarded at a higher rate than those made in daughters. Inequality (1) reflects the key ideas behind the TWH: (1) there is a benefit of improved maternal condition (neither $w_{\mathrm{m}, 1} / w_{\mathrm{m}, 2}$ nor $w_{\mathrm{f}, 1} / w_{\mathrm{f}, 2}$ is less than unity) and (2) the benefit of improved condition is felt more strongly by sons $\left(w_{\mathrm{m}, 1} / w_{\mathrm{m}, 2}\right.$ always exceeds $\left.w_{\mathrm{f}, 1} / w_{\mathrm{f}, 2}\right)$. Of course, condition may be due to things other than quality of breeding site, so our model is one of many possible Trivers-Willard scenarios.

\section{Inclusive Fitness Analysis \\ The Inclusive Fitness Effect}

The analysis of the model uses a version of Hamilton's (1964) notion of inclusive fitness, generalized to reflect the sex structure of the population. To capture the action of kin selection on the population average behavior $z_{i}$, we use $\Delta W_{i} \equiv \Delta W_{i}\left(z_{1}, z_{2}\right)$, the so-called inclusive fitness effect of $z_{i}$. When $\Delta W_{i}>0$, kin selection favors individuals who 
invest more than an average amount in sons, and $z_{i}$ increases. Alternatively, when $\Delta W_{i}<0$, kin selection favors individuals who invest more than an average amount in daughters, and $z_{i}$ decreases. A population is at an (internal) equilibrium with respect to the evolution of $z_{i}$ whenever $\Delta W_{i}=0$.

More formally, $\Delta W_{i}$ describes the marginal change in a mother's contribution to the gene pool of the population in the very distant future (i.e., her inclusive fitness), where the change itself is due to small changes in her own sex allocation behavior. A key quantity in the construction of $\Delta W_{i}$, then, will be per capita reproductive value (i.e., the probability that an allele chosen at random from a population of descendants originated from a given individual in the distant past).

In keeping with previous kin-selection models of sex ratio evolution (e.g., Taylor and Frank 1996; Wild and Taylor 2004), we split $\Delta W_{i}$ into two parts. The first part, called the "inclusive fitness effect through sons," reflects those inclusive fitness changes that occur when investment in sons is increased. The second part, the "inclusive fitness effect through daughters," reflects the inclusive fitness changes that occur when investment in daughters is increased. It is useful to note that the way in which we approach our kin-selection argument (below) differs from the recent "direct fitness" approaches used to study sex ratio evolution (e.g., Taylor and Frank 1996; Pen and Weissing 2002). The two approaches will, however, yield the same results (Taylor et al. 2007).

\section{The Inclusive Fitness Effect through Sons}

Fix attention on one mother (the focal mother) breeding on a type $i$ patch. Suppose that this focal mother increases the proportion of resources she gives to her male offspring by some small amount, $\delta_{\mathrm{m}}$. Suppose further that the increased investment in sons does not come at the expense of decreased investment in daughters (this is not an assumption per se, because changes in the level of investment in daughters are captured in "The Inclusive Fitness Effect through Daughters"). What are the inclusive fitness consequences of this type of deviant behavior?

To answer the question, we observe that the deviant mother has produced $K \delta_{\mathrm{m}}$ "extra" sons; each extra son contributes to the gene pool of future generations with a probability proportional to its reproductive value; only half of the alleles carried by the extra sons were contributed by the deviant mother herself. Putting these observations together allows us to express the inclusive fitness effect of a son born on a type $i$ patch as

$$
\Delta W_{\mathrm{m}, i}=\frac{1}{2} K \delta_{\mathrm{m}} v_{\mathrm{m}, i}
$$

where $v_{\mathrm{m}, i}$ is the reproductive value of a male born on a type $i$ patch (measured at the beginning of stage 2 in the life cycle).

Since the total reproductive value of males (and indeed females) as a group is conserved (Fisher 1930), the extra reproductive value created by deviant behavior must displace an equal amount of existing reproductive value in the population. However, we assume that complete male dispersal implies that the focal mother has no genetic stake in the reproductive value that is displaced. Consequently, displaced reproductive value does not enter into our $\Delta W_{\mathrm{m}, i}$ calculation.

\section{The Inclusive Fitness Effect through Daughters}

Now instead suppose that the deviant mother increases investment in daughters by a small amount, $\delta_{\mathrm{f}}$ (without decreasing investment in sons). As above, the deviant behavior increases the inclusive fitness of the focal mother by an amount $1 / 2\left(K \delta_{\mathrm{f}} v_{\mathrm{f}, i}\right)$, where $v_{\mathrm{f}, i}$ is the reproductive value of a female born on a type $i$ patch (measured at the beginning of stage 2 in the life cycle). However, in contrast to the situation with sons, this increase is not the only inclusive fitness change that occurs.

The extra reproductive value created by increased investment in daughters must displace an equal amount of existing reproductive value from the population. Unlike males, females exhibit some degree of philopatry. Consequently, the focal mother does have a genetic stake in the reproductive value that gets displaced. The actual amount of this genetic stake, though, is not immediately clear.

To calculate the reduction in inclusive fitness due to increased investment in daughters, we first note that we can write

$$
v_{\mathrm{f}, i}=\underbrace{(1-d) v_{f,(i, i)}^{\prime}}_{\text {local }}+d \sum_{j} p_{j} v_{\mathrm{f},(i, j)}^{\prime},
$$

where $v_{f,(i, j)}^{\prime}$ is the reproductive value of a female born on a type $i$ patch and competing on a type $j$ patch (measured at the end of stage 3 of the life cycle). In words, equation (3) tells us that the reproductive value of a female before dispersal is a weighted average of her reproductive value given that she does not disperse and her reproductive value given that she does disperse.

It is the first term of equation (3)-labeled "local" that counts toward a decrease in inclusive fitness for the focal mother. Note, however, that the displaced repro- 
ductive value only counts toward a reduction in inclusive fitness in the event that the local breeding site would have been won by another of the daughters produced by the focal mother. Since $\delta_{\mathrm{f}}$ is small, we can approximate the probability of this event with

$$
r_{i}=\frac{K(1-d) w_{\mathrm{f}, i}\left(1-z_{i}\right)}{N_{\mathrm{f}, i}}
$$

The coefficient $r_{i}$ can be used to measure the strength of LRC on a type $i$ breeding patch. The effect of LRC is its strongest when $r_{i}=1$, and LRC is absent when $r_{i}=0$.

The total reduction in inclusive fitness due to LRC can now be expressed as $1 / 2\left[K \delta_{\mathrm{f}}(1-d) v_{\mathrm{f},(i, i)}^{\prime} r_{i}\right]$. In turn, the overall inclusive fitness effect through daughters is

$$
\begin{aligned}
\Delta W_{f, i} & =\text { increase }- \text { decrease } \\
& =\frac{1}{2} K \delta_{\mathrm{f}}\left[v_{\mathrm{f}, i}-(1-d) v_{\mathrm{f},(i, i)}^{\prime} r_{i}\right] .
\end{aligned}
$$

$$
\text { The Inclusive Fitness Effect ... Again }
$$

In reality, investment in sons does come at the expense of daughters and vice versa. It follows that $\delta_{\mathrm{m}}=-\delta_{\mathrm{f}}$. The net effect of increased investment in sons-what we call $\Delta W_{i}$ - can now be expressed as

$$
\begin{aligned}
\Delta W_{i} & =\left(\Delta W_{\mathrm{m}, i}+\Delta W_{\mathrm{f}, i}\right) \\
& \propto v_{\mathrm{m}, i}-\left[d \sum_{j} p_{j} v_{\mathrm{f},(i, j)}^{\prime}+(1-d) v_{f,(i, i)}^{\prime}\left(1-r_{i}\right)\right] .
\end{aligned}
$$

Explicit expressions for $v_{\mathrm{m}, i}$ and $v_{\mathrm{f},(i, j)}^{\prime}$ are presented in the appendixes.

\section{A Comment on Stability}

Discussions surrounding the stability of a trait (or in this case a pair of traits) is often facilitated by making explicit reference to some dynamic description of evolution. If we suppose that evolution proceeds slowly, so that one generation represents only an infinitesimal fraction of evolutionary time (denoted $t$ ), then it is reasonable to describe the action of selection with the continuous (evolutionary) time dynamic

$$
\frac{d}{d t}\left[\begin{array}{l}
z_{1} \\
z_{2}
\end{array}\right]=(\text { positive constant }) \times\left[\begin{array}{l}
\Delta W_{1}\left(z_{1}, z_{2}\right) \\
\Delta W_{2}\left(z_{1}, z_{2}\right)
\end{array}\right]
$$

where values of $z_{1}$ and $z_{2}$ are taken from the unit square.
Equation (6) is a simplified version of multidimensional evolutionary dynamics studied by other authors (e.g., Greenwood-Lee et al. 2001; Day and Taylor 2003). Our simplified evolutionary dynamic assumes that (1) the sex allocation behavior exhibited by an individual in a given condition is not constrained by the behavior the individual itself would have exhibited in the opposite condition, and (2) both conditional sex allocation behaviors exhibit the same amount of variation. Our investigation, then, steers clear of the issues surrounding the genetic constraints on the evolution of $z_{1}$ and $z_{2}$.

We consider a pair of strategies, $\left(z_{1}, z_{2}\right)=\left(Z_{1}, Z_{2}\right)$, to be stable provided the pair corresponds to a locally asymptotically stable steady state of (6). When both $Z_{1}$ and $Z_{2}$ occur inside of the unit square, that is, when $\Delta W_{1}\left(Z_{1}, Z_{2}\right)=0$ and $\Delta W_{2}\left(Z_{1}, Z_{2}\right)=0$, we require that the eigenvalues of the matrix

$$
\left[\begin{array}{ll}
\frac{\partial \Delta W_{1}}{\partial z_{1}} & \frac{\partial \Delta W_{1}}{\partial z_{2}} \\
\frac{\partial \Delta W_{2}}{\partial z_{1}} & \frac{\partial \Delta W_{2}}{\partial z_{2}}
\end{array}\right]_{z_{1}=Z_{1}, z_{2}=Z_{2}}
$$

have negative real parts. If both $Z_{1}$ and $Z_{2}$ occur on the boundary of phenotype space, then the pair $\left(Z_{1}, Z_{2}\right)$ is stable whenever

$$
\Delta W_{i}\left(Z_{1}, Z_{2}\right) \begin{cases}<0 & \text { if } Z_{i}=0 \\ >0 & \text { if } Z_{i}=1\end{cases}
$$

for both $i=1$ and $i=2$.

Suppose now that one of the two behaviors (say $Z_{j}$ ) occurs on the boundary of phenotype space and the other (say $Z_{k}$ ) does not. In this case, $\Delta W_{k}$ vanishes but $\Delta W_{j}$ does not. We will consider such a pair to be stable whenever

$$
\Delta W_{j}\left(Z_{1}, Z_{2}\right) \begin{cases}<0 & \text { if } Z_{j}=0 \\ >0 & \text { if } Z_{j}=1\end{cases}
$$

and whenever

$$
\left.\frac{\partial \Delta W_{k}}{\partial z_{k}}\right|_{z_{1}=Z_{1}, z_{2}=Z_{2}}<0
$$

\section{Results for Well-Known Special Cases}

\section{Local Resource Competition}

By setting female dispersal $d$ equal to zero in equation (5), we recover a version of the standard model used to describe the effect LRC has on the sex ratio (Clark 1978; 
Table 2: Stable sex allocation behaviors and sex ratio (no. females : males) for complete female dispersal $(d=1)$

\begin{tabular}{cccc}
\hline & \multicolumn{3}{c}{ Parameters } \\
\cline { 2 - 4 } & $\frac{p_{2}}{p_{1}}<\frac{w_{\mathrm{f}, 1}}{w_{\mathrm{f}, 2}}<\frac{w_{\mathrm{m}, 1}}{w_{\mathrm{m}, 2}}$ & $\frac{w_{\mathrm{f}, 1}}{w_{\mathrm{f}, 2}}<\frac{p_{2}}{p_{1}}<\frac{w_{\mathrm{m}, 1}}{w_{\mathrm{m}, 2}}$ & $\frac{\mathrm{w}_{\mathrm{f}, 1}}{\mathrm{w}_{\mathrm{f}, 2}}<\frac{w_{\mathrm{m}, 1}}{w_{\mathrm{m}, 2}}<\frac{p_{2}}{p_{1}}$ \\
\hline Stable $z_{1}$ & $\frac{1}{2}\left(1+\frac{p_{2} w_{\mathrm{f}, 2}}{p_{1} w_{\mathrm{f}, 1}}\right)$ & 1 & 1 \\
Stable $z_{2}$ & 0 & 0 & $\frac{1}{2}\left(1-\frac{p_{1} w_{\mathrm{m}, 1}}{p_{2} w_{\mathrm{m}, 2}}\right)$ \\
Sex ratio & $\frac{w_{\mathrm{f}, 1}+p_{2}\left(w_{\mathrm{f}, 1}-w_{\mathrm{f}, 2}\right)}{w_{\mathrm{f}, 1}-p_{2}\left(w_{\mathrm{f}, 1}-w_{\mathrm{f}, 2}\right)}$ & $\frac{p_{2}}{p_{1}}$ & $\frac{w_{\mathrm{m}, 2}+p_{1}\left(w_{\mathrm{m}, 1}-w_{\mathrm{m}, 2}\right)}{w_{\mathrm{m}, 2}-p_{1}\left(w_{\mathrm{m}, 1}-w_{\mathrm{m}, 2}\right)}$ \\
\hline
\end{tabular}

Note: Under complete female dispersal, our inclusive fitness analysis yields the same results as the population genetic models investigated by Charnov (1979) and Bull (1981). The table formally describes the conclusions of a verbal argument originally presented by Trivers and Willard (1973) for the case in which maternal condition is determined by the quality of her breeding patch.

Wild and Taylor 2005). In this case, $r_{i}=1$, and so from (5) we see that $\Delta W_{i} \propto v_{\mathrm{m}, i}$, which is always greater than 0 . It follows that selection always favors increased investment in sons and strongly male-biased population sex ratios. It should be emphasized that we are not making any additional assumptions about $w_{\mathrm{m}, 1} / w_{\mathrm{m}, 2}$ or $w_{\mathrm{f}, 1} / w_{\mathrm{f}, 2}$ here.

\section{Condition-Dependent Sex Allocation in a Well-Mixed Population: A Version of the TWH}

When there is complete dispersal by females, that is, when $d=1$, the inclusive fitness effect can be written as $\Delta W_{i} \propto v_{\mathrm{m}, i}-v_{\mathrm{f}, i}$, where now

$$
\begin{aligned}
v_{\mathrm{m}, i} & =\frac{w_{\mathrm{m}, i}}{K \sum_{j} w_{\mathrm{m}, j} p_{j} z_{j}}, \\
v_{\mathrm{f}, i} & =\frac{w_{\mathrm{f}, i}}{K \sum_{j} w_{\mathrm{f}, j} p_{j}\left(1-z_{j}\right)} .
\end{aligned}
$$

The assumption $w_{\mathrm{m}, 1} / w_{\mathrm{m}, 2}>w_{\mathrm{f}, 1} / w_{\mathrm{f}, 2}$ leads to a situation in which at least one of $z_{1}$ and $z_{2}$ is as extreme as possible, that is, a situation in which both types of mother do not simultaneously produce mixed-sex broods. In this case, stable investment behaviors $z_{1}$ and $z_{2}$ are always biased in opposite directions and stable population-wide sex ratios is never male biased (table 2, and see, e.g., fig. 1). In fact, except for the case $w_{\mathrm{f}, 1}=w_{\mathrm{f}, 2}$, the population sex ratio is biased toward females, providing a marked contrast to the LRC model explored above. These same results have been produced by the population genetic models of Charnov (1979) and Bull (1981), and so we will not discuss them further.

We will refer to the special case $d=1$ as a "Charnov-
Bull model," and we note that it is a formalization of the verbal theory outlined by Trivers and Willard (1973) for the case in which maternal condition is determined by the quality of the breeding patch and fitness returns on pa-

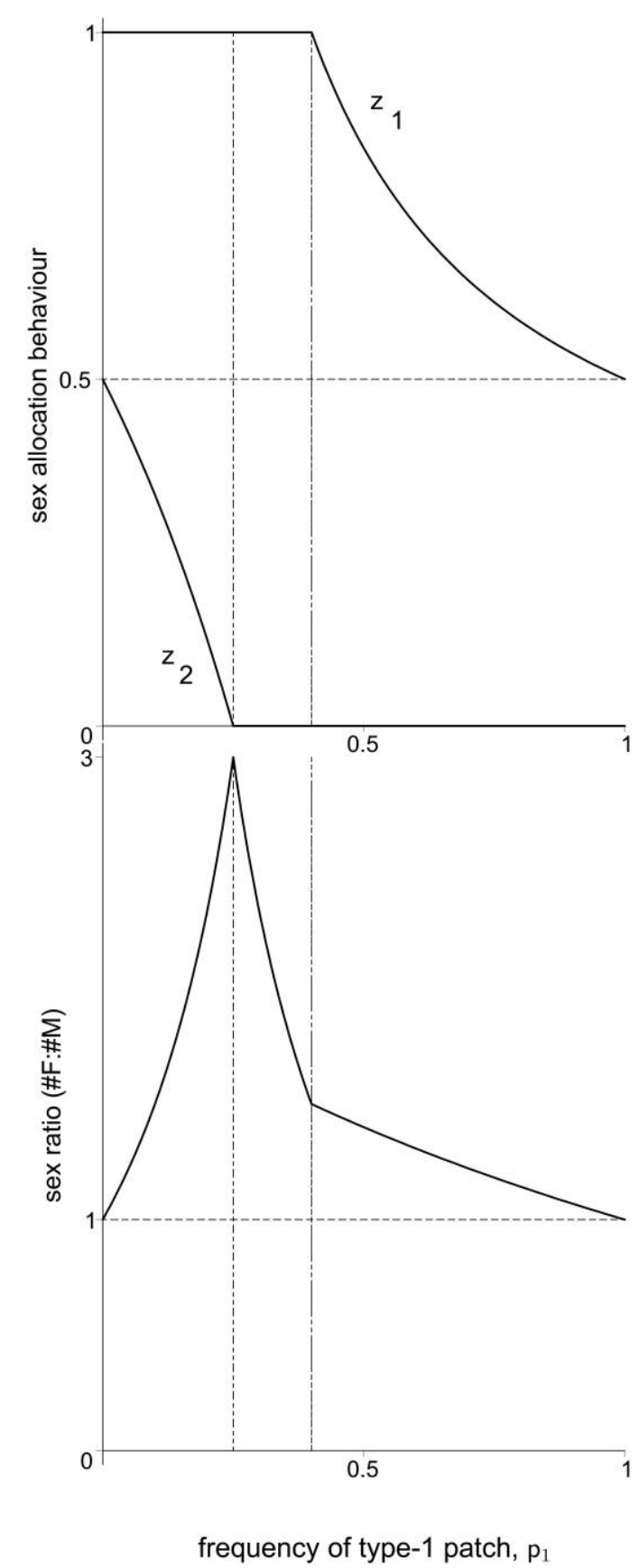

Figure 1: Stable sex allocation behavior and population-wide sex ratios, no. females : no. males (and labeled simply "sex ratios"), as a function of $p_{1}$ for the case $d=1, w_{\mathrm{m}, 1} / w_{\mathrm{m}, 2}=3$, and $w_{\mathrm{m}, 1} / w_{\mathrm{m}, 2}=1.5$. The figure illustrates results already presented in table 2 but provides a visual reference for results presented in figures 3 and 4 . 
rental investment are linear (cf. more general return functions considered by Frank [1987]). Recall that the assumption $w_{\mathrm{m}, 1} / w_{\mathrm{m}, 2}>w_{\mathrm{f}, 1} / w_{\mathrm{f}, 2}$ means that the investments made in sons by mothers in good condition (i.e., mothers breeding on a high-quality patch) are always rewarded at a higher rate than those made in daughters. As Trivers and Willard (1973) predicted, we see that mothers in good condition tend to produce more sons than daughters (table 2). (Trivers and Willard [1973] made no specific predictions about population sex ratios.)

\section{Combining LRC and Condition-Dependent Allocation}

\section{Overview}

Local resource competition and the adjustment of parental investment strategies in response to changes in breeding condition are leading explanations for the adaptive significance of sex allocation behavior and population sex ratios of many vertebrate species (Cockburn et al. 2002). On one hand, local resource competition in a homogeneous environment encourages the evolution of malebiased sex allocation behavior as well as male-biased population sex ratios ("Local Resource Competition"). On the other hand, a simple, well-mixed but heterogeneous environment (and the associated variablity in maternal condition) encourages the evolution of extreme conditional investment strategies biased in opposite directions and population sex ratios that are mainly biased toward females ("Condition-Dependent Sex Allocation in a Well-Mixed Population: A Version of the TWH”). Combining the two approaches is thought to lead to tremendous complications (e.g., Cockburn et al. 2002)

In this section we show that in some nontrivial cases, models of condition-dependent sex allocation that account for LRC make the same predictions as models that ignore LRC. In other cases we see that it is possible for LRC to complicate predictions about condition-dependent sex allocation substantially. Furthermore, we present numerical results that suggest LRC is a significant complication in those circumstances in which there is little difference between the advantage enjoyed by sons and daughters born to mothers in good condition. The section ends with a brief investigation into how the strength of LRC varies across a population at evolutionary equilibrium.

\section{No Broods of Mixed Sex}

We begin the analysis of the general model by restricting attention to those populations in which each fertilized female produces either only sons or only daughters. Such populations are said to exhibit "joint extreme investment behavior" and are especially convenient to work with be- cause the total reproductive value of type 1 parents can be related in a simple way to the total reproductive value of type 2 parents.

Mathematically, we suppose that population average strategies are either

$$
z_{1}=1, z_{2}=0
$$

or

$$
z_{1}=0, z_{2}=1
$$

The calculations presented in appendix B show that in the presence of LRC, (7) is stable whenever

$$
\frac{w_{\mathrm{m}, 1}}{w_{\mathrm{m}, 2}}>\frac{p_{2}}{p_{1}} \times\left\{\frac{2\left(1-d p_{1}\right)^{2}}{2 d p_{2}\left[d p_{2}+2(1-d)\right]+(1-d)^{2}}\right\}
$$

and

$$
\frac{w_{\mathrm{f}, 1}}{w_{\mathrm{f}, 2}}<\frac{p_{2}}{p_{1}} \times\left[\frac{2 d p_{1}\left(1-d p_{1}\right)}{1-d\left(1-2 d p_{1} p_{2}\right)}\right]
$$

In words, stability of the pair of behaviors in (7) is assured whenever (1) the relative competitive ability of sons born to mothers in good condition is large enough to discourage the production of sons by mothers in poor condition (inequality [9]), and (2) the relative competitive ability of any daughters born on a type 1 patch is modest enough to discourage the production of daughters by those mothers currently investing only in sons (inequality [10]).

In order for inequality [10] to hold, the frequency of type 1 patches cannot exceed one-half (fig. $2 A$ ). This means that the stability condition (10) implies a female-biased sex ratio $p_{2} / p_{1}>1$. This leads us to the first main conclusion of our analysis.

Conclusion 1. Under a reasonable set of conditions, the conclusions about sex allocation behavior and populationwide sex ratios-made under assumptions consistent with those of Trivers and Willard (1973)_-are not complicated by local resource competition.

A similar analysis of the case in which $z_{1}=0$ and $z_{2}=1$ (eqq. [8]) shows us that such conditional behaviors are stable whenever

$$
\frac{w_{\mathrm{m}, 1}}{w_{\mathrm{m}, 2}}<\frac{p_{2}}{p_{1}} \times\left\{\frac{2 d p_{1}\left[d p_{1}+2(1-d)\right]+(1-d)^{2}}{2\left(1-d p_{2}\right)^{2}}\right\}
$$




$$
\frac{w_{\mathrm{f}, 1}}{w_{\mathrm{f}, 2}}>\frac{p_{2}}{p_{1}} \times\left[\frac{1-d\left(1-2 d p_{1} p_{2}\right)}{2 d p_{2}\left(1-d p_{2}\right)}\right]
$$

In this case, stability of the pair $z_{1}=0$ and $z_{2}=1$ in (8) is assured because (1) the relative competitive ability of any sons born to on a high-quality patch is not strong
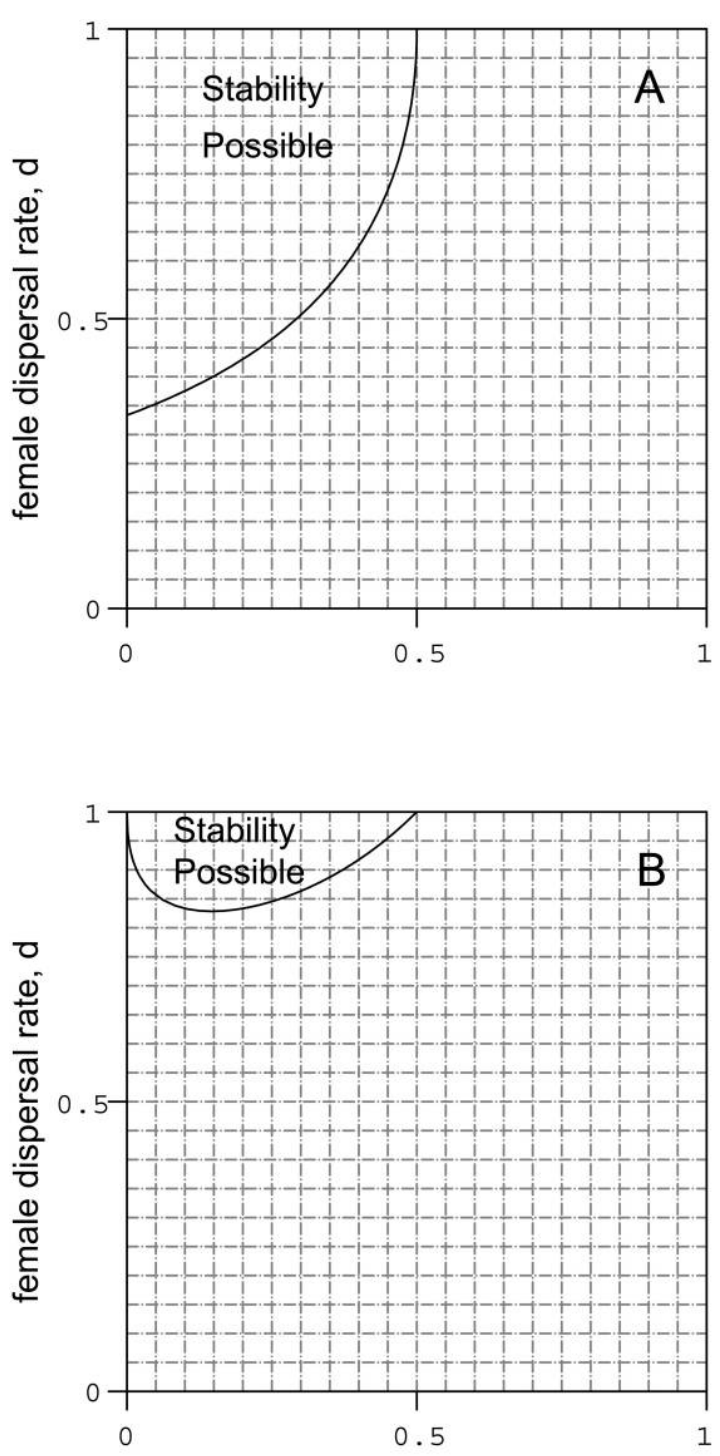

frequency of type-1 patch, $\mathrm{p}_{1}$

Figure 2: Regions of $d-p_{1}$ parameter space in which it is possible for either condition (10)-for the stability of $z_{1}=1, z_{2}=0$-to hold $(A)$ or for condition (12) -for the stability of $z_{1}=0, z_{2}=1$-to hold $(B)$. In both panels, stability conditions imply that pairs $\left(d, p_{1}\right)$ fall into the region above the curve. Although the regions indicated in $A$ and $B$ overlap, we can rule out the possibility of bistability. enough to discourage the production of daughters by mothers in good condition (inequality [11]), and (2) the relative competitive ability of daughters born to mothers in good condition is large enough to discourage the production of daughters by mothers in poor condition (inequality [12]). It is interesting to note that $z_{1}=0$ can be part of a stable pair of conditional investment strategies when $w_{\mathrm{m}, 1} / w_{\mathrm{m}, 2}>w_{\mathrm{f}, 1} / w_{\mathrm{f}, 2}$ because this possibility can only hold when the aforementioned inequality is reversed in the absence of LRC (Charnov 1979; Bull 1981).

In order for inequality [11] to hold, the frequency of type 1 patches must not exceed one-half (fig. $2 B$ ). Unlike the previous case, the appropriate stability condition, condition [11], now implies a male-biased sex ratio, $p_{1} / p_{2}<$ 1. Again, this provides a stark contrast to the predictions made by the Charnov-Bull model.

Conclusion 2. Our second main conclusion can now be stated as follows: LRC can greatly complicate predictions about sex allocation behavior and population sex ratios. In some cases (e.g., above), our model leads to predictions that are qualitatively opposite to those made by models that ignore LRC.

\section{Numerical Investigations}

We have found that the model is analytically tractable for any case in which only one type of mother invests in daughters (not just those cases explored above). Nevertheless, we forgo such analysis in favor of a numerical investigation. Our numerical investigation tracks how, for fixed advantages $w_{\mathrm{m}, 1} / w_{\mathrm{m}, 2}$ and $w_{\mathrm{f}, 1} / w_{\mathrm{f}, 2}$, the stable levels of sex allocation behavior change as we change $d$ and $p_{1}$. In technical terms, we use numerical methods (implemented with the Maple software package) to investigate structural stability properties of the model in general.

Figure 3 shows the results of numerical investigations. As expected, we see the predictions of LRC models indicated by a green curve of the face, $d=0$, and the predictions of the Charnov-Bull models indicated by a blue curve on the face, $d=1$ (cf. fig. 1).

By increasing $d$ from 0 to a small positive value, we move into a region of parameter space in which both types of mother produce mixed-sex broods (fig. 3, black curves). Joint production of mixed-sex broods is a phenomenon that does not occur in the Charnov-Bull models, and it also does not occur in the version of the LRC model we developed above ("Local Resource Competition").

Increasing $d$ further leads to levels of sex allocation behavior that are qualitatively consistent with the sex allocation predictions made by the Charnov-Bull models (fig. 3 , red curves). That is to say, red curves indicate those stable pairs of strategies in which $z_{1}$ is male biased, $z_{2}$ is 

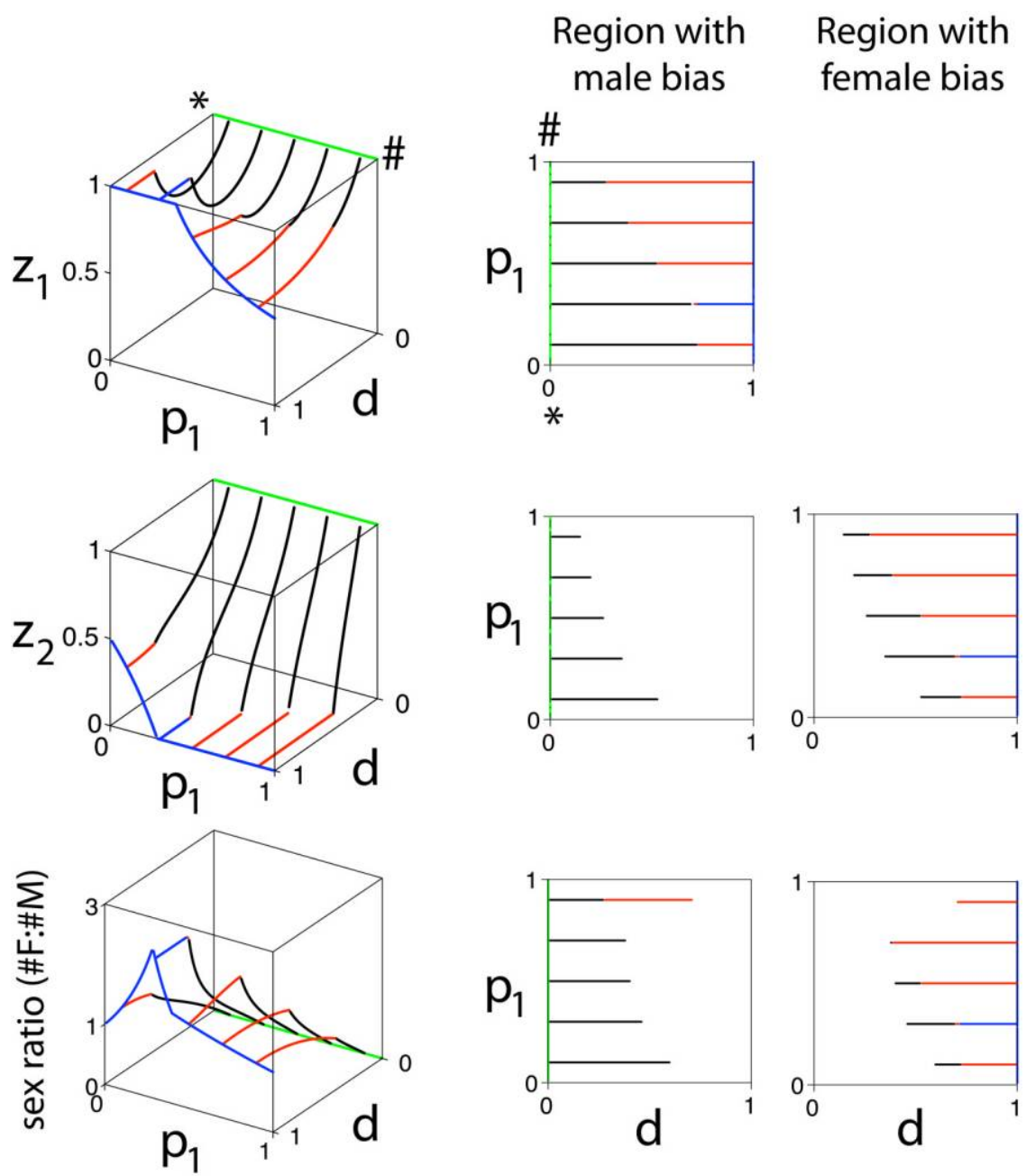

Figure 3: Stable sex allocation behaviors and population sex ratios (labeled simply "sex ratios") as $d$ and $p_{1}$ change. In this case, $w_{\mathrm{m}, 1} / w_{\mathrm{m}, 2}=2.5$ and $w_{\mathrm{f}, 1} / w_{\mathrm{f}, 2}=1.5$; that is, male advantage was about $67 \%$ greater than female advantage. Panels on the far left show a transition between sex allocation predictions made by LRC models (green) to those made by standard models of condition-dependent sex allocation behavior (blue). Notice the similarity between the blue curves and those presented in figure 1. Black curves indicate those situations in which both types of breeding female produce broods of mixed sex. Red curves indicate situations in which sex allocation behaviors (but not necessarily sex ratio) are qualitatively consistent with standard models of Charnov (1979) and Bull (1981): type 1 females bias investment toward sons, type 2 females bias investment toward daughters, and at least one sex allocation behavior is as extreme as possible. Smaller panels to the right give the top view of the $d$ - $p_{\text {, }}$ plane and highlight those points where sex allocation behaviors and sex ratios are, respectively, male biased (center) or female biased (far right). To help orient the reader, we have included two landmarks: the number sign and the asterisk.

female biased, and only one of $z_{1}$ or $z_{2}$ is as extreme as possible. Red curves do not necessarily indicate population-wide female bias; although sex allocation behaviors are qualitatively consistent with Charnov-Bull results, the population-wide sex ratio bias might not be.

As $d$ approaches 1, we also find visual evidence of the argument already made in "No Broods of Mixed Sex." That is to say, we recover a model that allows for LRC while making the same predictions as the Charnov-Bull models (fig. 2, blue curve not on face $d=1$ ).
In sum, figure 3 illustrates a gradual transition between the predictions models of LRC $(d=0)$ and those of Charnov-Bull $(d=1)$. However, there are cases in which the transition between the different predictions is abrupt. Figure 4 illustrates one such case.

In figure 4 we see again that increasing $d$ from 0 to a small positive value leads to the production of mixed-sex broods by both type 1 and type 2 mothers (fig. 4, black curves). Increasing $d$ further leads to the (arguably) counterintuitive situation in which low-quality mothers invest 

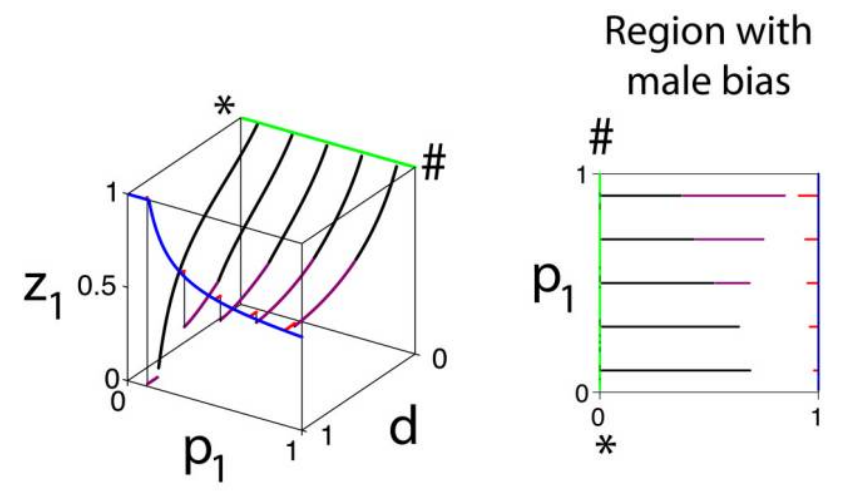

\section{Region with female bias}
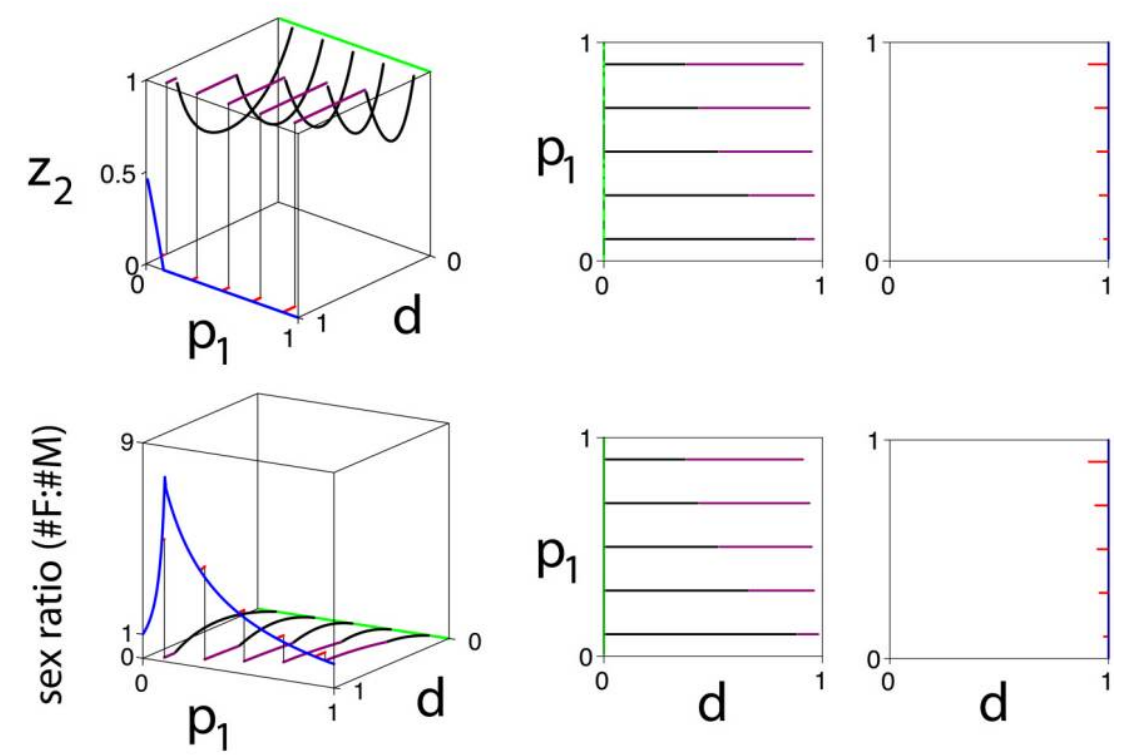

Figure 4: Stable sex allocation behaviors and population sex ratios (labeled simply "sex ratios") as $d$ and $p_{1}$ change. In this case, $w_{\mathrm{m}, 1} / w_{\mathrm{m}, 2}=7.7$ and $w_{\mathrm{f}, 1} / w_{\mathrm{f}, 2}=7.2$; that is, male advantage was only $7 \%$ greater than female advantage, in contrast to figure 3. Panels on the far left show a transition between sex allocation predictions made by LRC models (green) to those made by standard models of condition-dependent sex allocation behavior (blue). Thick black curves indicate those situations in which both types of breeding female produce broods of mixed sex. Red curves indicate situations in which sex allocation behaviors (but not necessarily sex ratio) are qualitatively consistent with standard models of Charnov (1979) and Bull (1981): type 1 females bias investment toward sons, type 2 females bias investment toward daughters, and at least one sex allocation behavior is as extreme as possible. Purple curves indicate situations in which at least one condition sex allocation behavior is as extreme as possible, and bias in sex allocation behavior is qualitatively inconsistent with the predictions made by models of Charnov (1979) and Bull (1981). Smaller panels to the right give the top view of the $d-p_{1}$ plane and highlight those points where sex allocation behaviors and sex ratios are, respectively, male biased (center) or female biased (far right). To help orient the reader, we have included two landmarks: the number sign and the asterisk.

completely in sons, and high-quality mothers either invest in both sons and daughters or simply invest in daughters (purple curves). We expect the latter situation to occur whenever inequalities (11) and (12) hold.

Remarkably, we see that the main qualitative predictions of LRC models-namely, male-biased sex allocation behaviors and male-biased population sex ratios-can be maintained even under high rates of female dispersal (fig. 4). We can, of course, increase $d$ to the point at which model predictions qualitatively match those of the Char-
nov-Bull models (red curves). Now, however, the transition between the different qualitative preditions occurs abruptly. (The transition itself is characterized by the existence of a line of stable sex allocation behaviors and population sex ratios [thin vertical black lines].) Numerical results presented in this section lead us to suggest the next conclusion.

Conclusion 3. The qualitative predictions of previous models that ignore LRC are extremely sensitive to the pres- 
ence of incomplete female dispersal when the difference between the advantage enjoyed by sons born to a mother in good condition and that enjoyed by their sisters (measured relative to sister's advantage) is not sufficiently large. Unfortunately, the numerical results do not tell us, a priori, how large "sufficiently large" is.

\section{Variation in the Strength of Local Resource Competition}

If both types of mother invest in daughters, then both incur inclusive fitness losses due to local competition. It has been suggested that such losses are less for parents breeding on high-quality patches (Silk 1983). Of course, reduced local resource competition on high-quality patches could be due to certain habitat-specific patterns of female dispersal. Our model does not allow habitatspecific dispersal. Despite this simplification, we see that reduced local resource competition on high-quality patches can emerge from the action of selection on the sex ratio alone, though some exceptions exist (table 3 ).

\section{Discussion}

Theoretical Predictions

We have examined the consequences of combining the standard assumptions of (1) LRC models of sex allocation (e.g., Clark 1978) and (2) models describing the evolution of condition-dependent sex allocation (Trivers and Willard 1973). Given that the models that examine these factors separately make qualitatively different predictions (Clark 1978; Charnov 1979; Bull 1981; Wild and Taylor 2005), we might expect that combining them would lead to a gradual transition between the different predictions as we move through parameter space (in particular, as $d$ changes). The most striking result we present here is that in general, such intuition fails.

If conditions (9) and (10) hold, then the predictions made by Trivers and Willard hold: females should be produced on low-quality patches and males on high-quality patches. In these cases, and from the standpoint of qualitative predictions, LRC can be effectively ignored. Our numerical results suggest that LRC can even be overlooked by verbal arguments in some cases in which (9) and/or (10) fail, provided the advantage given to males born on high-quality patches is sufficiently greater than that given to females born on high-quality patches. Of course, female philopatry can be adjusted so that model predictions coincide instead with those made by LRC models of sex ratio evolution, but as long as $w_{\mathrm{m}, 1} / w_{\mathrm{m}, 2}$ is sufficiently larger than $w_{\mathrm{f}, 1} / w_{\mathrm{f}, 2}$, there appears to be a gradual transition between the two extremes.

In contrast, we sometimes obtain predictions exactly
Table 3: Comparison of the strength of local resource competition (LRC) on different patches for selected numerical examples

\begin{tabular}{|c|c|c|c|c|c|c|c|}
\hline \multicolumn{4}{|c|}{ Parameters } & \multicolumn{2}{|c|}{$\begin{array}{c}\text { Stable } \\
\text { behaviors }\end{array}$} & \multicolumn{2}{|c|}{$\begin{array}{l}\text { Strength of } \\
\text { LRC }\end{array}$} \\
\hline$w_{\mathrm{m}, 1} / w_{\mathrm{m}, 2}$ & $w_{\mathrm{f}, 1} / w_{\mathrm{f}, 2}$ & $d$ & $p_{1}$ & $z_{1}$ & $z_{2}$ & $r_{1}$ & $r_{2}$ \\
\hline \multirow[t]{3}{*}{4.0} & 2.1 & .7 & .6 & .6536 & 0 & .2715 & .3388 \\
\hline & & .2 & .9 & .7478 & 0 & .7860 & .8740 \\
\hline & & .1 & .1 & .8611 & 0 & .7386 & .9064 \\
\hline \multirow[t]{3}{*}{2.2} & 2.1 & .7 & .6 & .4778 & .7044 & .3771 & .5205 \\
\hline & & .2 & .9 & .7408 & .6737 & .8064 & .7141 \\
\hline & & .1 & .1 & .8812 & .8339 & .9279 & .8955 \\
\hline
\end{tabular}

opposite to those made by the TWH: we find female-biased brood sex ratios on high-quality patches and male-biased brood sex ratios on low-quality patches in cases where the advantage given to males born on high-quality patches is not appreciably greater than that given to females born on high-quality patches. Remarkably, we also see that the gradual transition between the predictions of Trivers and Willard and those made by LRC models is lost (fig. 4). Simply put, the predictions of verbal arguments, like those made by Trivers and Willard, appear to be extremely sensitive to the presence of incomplete female dispersal when sons born to good mothers do not enjoy an intrasexual competitive advantage that is markedly greater than that enjoyed by their sisters.

Clearly, when parental condition is tied to the quality of a breeding patch, female philopatry does more than simply force us to consider the detrimental impact LRC has on inclusive fitness of a parent. Female philopatry also means that daughters born on high-quality patches are always more likely than their brothers to compete on a high-quality patch. This situation is analogous to one considered by Leimar (1996) in which daughters (not sons) inherit maternal condition. Leimar's model also predicts the possibility that mothers in good condition may prefer to invest in daughters rather than sons. In our model, incomplete female dispersal establishes an asymmetric pattern of "inheritance" of maternal condition by daughters. This inheritance increases the relative inclusive fitness return from producing daughters on high-quality patches because it increases the probability that descendents continue to breed on high-quality patches. When this inheritance effect is large enough, relative to increased mating success of high-quality sons, then we predict the opposite pattern to that suggested by TWH: females on high-quality patches should produce daughters.

\section{Applying to the Real World}

Our results have four clear implications for empirical tests of sex allocation theory. First, we have shown that when 
LRC and maternal condition influence sex allocation strategies, the population sex ratio can be biased in either direction, depending on the value of several parameters in our model (figs. 3,4 ). This is without even considering the complications that can be raised by differential mortality. Consequently, attempts to test theory based on whether the population sex ratio of a particular species are male or female biased will generally be misguided (Frank 1990; Pen and Weissing 2000a; West and Sheldon 2002; Wild 2006). This contrasts with what often appears to be conventual wisdom in the empirical literature, where it is often assumed that testing whether a population sex ratio is biased or not is testing for whether sex ratio adjustment is occurring (e.g., Palmer 2000). A notable exception to this is the application of the Trivers-Willard effect to sex-changing animals, where clear predictions can be made for the population sex ratio, as has recently been discussed in detail elsewhere (Allsop and West 2004).

Second, while it is extremely hard to make absolute predictions for the population sex ratio of a particular species, it is possible to make relative predictions for how the population sex ratio should vary across populations or species. For example, results in figures 3 and 4 suggest (1) a greater tendency for population sex ratios to be more male biased (or less female biased) in populations or species in which female philopatry is more prevalent and (2) a lesser tendency toward male-biased population sex ratios in populations or species in which the advantage of being a higher-quality offspring is relatively greater for males. The key point is that these predictions hold, even if both LRC and the assumptions of Trivers and Willard are operating. Support for the first of these predictions has already been found in primates and marsupials. Johnson (1988) found that across primate species, the sex ratio was more male biased in species in which there was greater competition for resources or lower rates of dispersal by females. Johnson et al. (2001) found that across populations of the common brushtail possum, the sex ratio was more male biased in populations in which there was greater competition between females for den sites.

Third, depending on parameter values, our model is able to predict conditional sex ratio adjustment in either direction, with females on high-quality patches producing a higher proportion of either sons or daughters. Consequently, the original Trivers-Willard prediction only holds under certain conditions. Leimar (1996) has previously shown that this problem arises if there is maternal transmission of quality to daughters through factors such as transmission of rank or inheritance of quality. This is because inheritance of maternal quality leads to high-quality mothers producing high-quality daughters who will produce high-quality offspring (grandchildren). Depending on parameter values, this can outweigh the fact that sons produced by high-quality mothers will have a higher reproductive success because they will not necessarily mate with high-quality females, and so this will not necessarily lead to high-quality grandchildren. The underlying tradeoff here is between producing more grandchildren (through high-quality sons) or higher-quality grandchildren (through high-quality daughters). This emphasizes that reproductive success (number of offspring) is not the same as reproductive value (which also accounts for differences in quality among offspring) and that it is the latter that matters to selection (Fisher 1930; Grafen 2006). Our model leads to this same issue for similar reasons, because daughters who do not disperse have a chance of inheriting their mothers' patches. The difference here is that inheritance of quality drops out of our model as a consequence of how we allow for LRC rather than as a consequence of an initial assumption. The relative importance of the original Trivers-Willard suggestion and maternal transmission could vary across taxa. For example, in ungulates, there is a general tendency for high-quality females to produce sons, suggesting that the Trivers-Willard effect is more important (Sheldon and West 2004), whereas in primates, a greater tendency for maternal transmission of rank to daughters may explain the lack of a consistent pattern of sex ratio adjustment (Brown and Silk 2002; West et al. 2005.

These results demonstrate the care that must be taken when testing for adaptive sex ratio adjustment in a species in response to maternal quality. The original TWH is only expected to hold under certain conditions. More specifically, in order to be able to predict the direction of sex ratio adjustment, it is necessary to know how maternal quality influences the reproductive value of offspring and not just their reproductive success (Leimar 1996). This can be hard because the direct measurement of the reproductive value of males and females produced by different quality females can require long-term multigeneration studies. Shorter-term studies will need to examine the different components of fitness separately. For example, the importance of maternal transmission of quality could be examined by investigating factors such as (1) temporal autocorrelation in patch success or (2) mother-daughter correlation in patch quality or identity. These issues could be addressed with observational or experimental (e.g., cross-fostering) methodologies. Alternatively, the effect of LRC and/or transmission of quality could be examined experimentally by manipulating density or dispersal. We hope that these issues do not make empirical workers too pessimistic-our aim is to emphasize empirical work that is needed rather than suggest things that are not worth doing. In particular, examining the underlying assumptions of sex allocation theory to demonstrate selection on sex ratio adjustment is as important as testing for sex ratio 
adjustment. The Cape mountain zebra provides an excellent candidate for further study because it appears to be a species in which there is strong maternal transmission of condition and selection for high-quality females to produce daughters (Lloyd and Rasa 1989).

Fourth, while it is very hard to predict what should happen in a particular species, it can be much easier to predict how the extent of sex ratio adjustment between individuals or patches should vary across populations or species. For example, the difference in the birth sex ratio of broods produced by high- and low-quality mothers, as determined by factors such as patch or maternal condition, should be greater when (1) the advantage of being a higher-quality offspring is relatively greater for males or (2) the extent of LRC is lower. There is support for these predictions from primates and ungulates. Both ungulates and primates show greater sex ratio shifts with maternal condition in species in which there is greater sexual dimorphism and hence stronger sexual selection for high male quality (Schino 2004; Sheldon and West 2004). In primates, the extent of sex ratio adjustment with maternal condition is lower in species in which there is likely to be greater LRC, as estimated from the population growth rate (van Schaik and Hrdy 1991; Schino 2004). If individuals can assess variation in the relative importance of LRC, then our model would also predict variation in the pattern of sex ratio adjustment within a species, either across populations or over time. Consistent with this, as the population density increased in a population of red deer, which could increase the importance of LRC, the extent to which high-quality females preferentially produced sons was reduced (Kruuk et al. 1999). In contrast, across populations of wild savannah baboons, the pattern of sex ratio adjustment did not vary consistently with population growth rate, another possible indicator of the extent of LRC (Silk et al. 2005). More generally, this emphasizes the power of using relative or comparative predictions to test evolutionary theory: it is often easier to make predictions for how traits should vary across individuals or populations or species than it is to predict their absolute value (Frank 1998; Griffin et al. 2005).

Our results also suggest a potential reason for why the pattern of sex ratio adjustment in vertebrates is usually not very extreme. A major problem for sex allocation theory is that in cases in which the birth sex ratio is expected to be adjusted in response to environmental conditions (as predicted by Trivers and Willard), the observed pattern is generally a gradual shift in response to environmental quality (e.g., maternal condition), whereas theory predicts a threshold shift from all male to all female at a critical value of the environmental value (e.g., at a certain host condition; West et al. 2002). We have shown that as LRC is introduced into the TWH through lower female dis- persal rates, the pattern of sex ratio adjustment across patches changes from producing only daughters in lowquality patches and only sons in high-quality patches to producing both sons and daughters in both low- and highquality patches. Previous theory has found it impossible to predict such gradual shifts in sex ratio unless extreme assumptions were made, such as the cost of sex ratio adjustment increasing exponentially the further one is from 50\% males (Charnov et al. 1981; Leimar 1996; Pen et al. 1999; Pen and Weissing 2002). More generally, this seems to be an area in which theory is falling behind empirical progress, as there is an expanding literature examining what factors correlate with the extent of sex ratio adjustment but an almost complete lack of theory able to predict such variation (e.g., van Schaik and Hrdy 1991; West and Sheldon 2002; Cameron 2004; Schino 2004; Sheldon and West 2004; Griffin et al. 2005; West et al. 2005).

\section{Challenges}

Vertebrate natural history suggests a number of directions in which it would be useful to develop further theory. In particular, there are several possibilities for even more complicated interactions between different selective forces. For example, considering LRC and the TWH, (1) in species with overlapping generations, there can be LRC between generations, the importance of which can vary with factors such as age or survival rates (Cockburn et al. 1985; Isaac et al. 2005); (2) the importance of LRC could co-vary with maternal (or patch) quality (Silk 1983); (3) there can be coevolution between dispersal rates and such allocation (Leturque and Rousset 2003, 2004; Wild and Taylor 2004; Wild et al. 2006); (4) there can also be cooperative interactions between relatives (local resource enhancement [LRE]) in populations in which LRC and the TWH occur (Komdeur et al. 1997; Komdeur 1998); and (5) the relative costs of raising a son or daughter can vary with maternal quality (Gomendio et al. 1990). In all cases, the interplay between theory and empirical research will be key, as empirical studies suggest the complications that can be ignored as well as those that need to be added. For example, we have assumed that related males do not compete with one another for access to females, as such local mate competition (LMC) is not important in vertebrates (West et al. 2005). However, the interplay of the TWH with LMC could be important in some parasitoid wasps, as has been explored elsewhere (e.g., Werren 1984; Werren and Simbolotti 1989; Ikawa et al. 1993).

Another major complication that requires further theoretical attention relates to how offspring quality is determined. We have assumed that the breeding condition of a mother is determined solely by the quality of patch on which she breeds. In reality, though, condition could 
be a quality that is passed from mother to offspring, independent of any dispersal "decision" made by the offspring itself (Leimar 1996). To complicate matters further, offspring could also inherit aspects of paternal condition (Pen and Weissing 2000b), leading to complicated sex allocation reaction norms. Modeling intricate transmission pathways is a difficult task, at least with a kin-selection approach. The main challenge lies in simply understanding the population dynamics of a system with both maternal and paternal input into offspring condition. The dynamics of such a system are inherently nonlinear, and it is not at all clear when these lead to a steady state distribution of conditions. Steady state distributions like these are critical to the formulation of any kin-selection model (Taylor
1990; Taylor and Frank 1996). A possible solution to this problem is to use a population genetic or quantitative genetic approach (Kirkpatrick and Lande 1989; Courteau and Lessard 2000).

\section{Acknowledgments}

G.W. is funded through the Natural Sciences and Engineering Research Council of Canada and through the Ontario Ministry of Science. S.A.W. thanks the Royal Society and the Natural Environment Research Council for funding. Comments from A. Gardner, B. Sheldon, J. Silk, L. Wahl, and two anonymous reviewers helped improve the original draft of this article.

\section{APPENDIX A}

\section{Calculation of Reproductive Value}

In this appendix we calculate $v_{\mathrm{f},(i, j)}^{\prime}$, the reproductive value of a female born on a type $i$ patch but competing on a type $j$ patch. We also calculate $v_{\mathrm{m},(i, j)}^{\prime}$, the reproductive value of a male born on a type $i$ patch but competing on a type $j$ patch. Both $v_{\mathrm{f},(i, j)}^{\prime}$ and $v_{\mathrm{m},(i, j)}^{\prime}$ can be expressed in terms of $V_{j}$, the reproductive value of an adult (male or female) breeding on a type $j$ patch. Consequently, our first step will be to calculate $V_{j}$ 's.

We will say that an allele is in state $i$ if it currently resides in an individual (male or female) breeding on a type $i$ patch. Let $P_{i j}$ denote the $j \rightarrow i$ transition probability, that is, the probability that an allele, currently in state $i$, was in state $j$ one generation ago. Transition probabilities can be summarized with a $2 \times 2$ matrix, $\mathbf{P}$. Let

$$
Q_{\mathrm{m}}=\frac{K p_{1} w_{\mathrm{m}, 1}}{N_{\mathrm{m}}}
$$

denote the probability that a male born on a type 1 patch competes successfully on any patch. Similarly, let

$$
Q_{\mathrm{f}, i}=\frac{K\left(1-d+d p_{i}\right) w_{\mathrm{f}, i}}{N_{\mathrm{f}, i}}
$$

denote the probability that a female born on at type $i$ patch also competes successfully on a type $i$ patch. Using this notation we can write

$$
\mathbf{P}=\frac{1}{2}\left[\begin{array}{cc}
Q_{\mathrm{f}, 1}+Q_{\mathrm{m}} & \left(1-Q_{\mathrm{f}, 1}\right)+\left(1-Q_{\mathrm{m}}\right) \\
\left(1-Q_{\mathrm{f}, 2}\right)+Q_{\mathrm{m}} & Q_{\mathrm{f}, 2}+\left(1-Q_{\mathrm{m}}\right)
\end{array}\right] .
$$

The $j$ th element of the dominant left eigenvector of $\mathbf{P}$ gives us the total reproductive value of type $j$ breeding adults (Taylor 1996). Let $c_{j}$ denote the $j$ th element of the dominant left eigenvector of $\mathbf{P}$. An elementary calculation yields

$$
\left[c_{1}, c_{2}\right] \propto\left[\left(1-Q_{\mathrm{f}, 2}\right)+Q_{\mathrm{m}},\left(1-Q_{\mathrm{f}, 1}\right)+\left(1-Q_{\mathrm{m}}\right)\right] .
$$

We calculate $V_{j}$ by dividing $c_{j}$ by frequency of individuals breeding on a type $j$ patch (Taylor 1996). This gives us

$$
\left[V_{1}, V_{2}\right] \propto\left[\frac{\left(1-Q_{\mathrm{f}, 2}\right)+Q_{\mathrm{m}}}{p_{1}}, \frac{\left(1-Q_{\mathrm{f}, 1}\right)+\left(1-Q_{\mathrm{m}}\right)}{p_{2}}\right]
$$


Finally, we express $v_{\mathrm{f},(i, j)}^{\prime}=$ probability of becoming breeding adult $\times$ reproductive value as breeding adult $=$ $\left(w_{\mathrm{f}, i} / N_{\mathrm{f}, j}\right) \times V_{j}$ and $v_{\mathrm{m},(i, j)}^{\prime}=$ probability of becoming breeding adult $\times$ reproductive value as breeding adult $=$ $\left(w_{\mathrm{m}, i} / N_{\mathrm{m}, j}\right) \times V_{j}$

\section{APPENDIX B}

\section{Populations with No Mixed-Sex Broods}

In this appendix we consider a population in which no mixed-sex broods are produced; that is, we suppose that

$$
z_{i}=1, z_{k}=0(i \neq k)
$$

In this case, type $i$ breeding females produce only sons, whereas type $k$ breeding females produce only daughters.

When (B1) holds, we have manageable expressions for the various reproductive values used in the inclusive fitness calculation (5):

$$
v_{\mathrm{m}, j}=\frac{w_{\mathrm{m}, j}}{N_{\mathrm{m}}}=\frac{w_{\mathrm{m}, j}}{K w_{\mathrm{m}, i} p_{i}}(j=i, k)
$$

and

$$
\begin{aligned}
& v_{\mathrm{f},(j, i)}^{\prime}=\frac{1}{2} \frac{w_{\mathrm{f}, j}}{N_{\mathrm{f}, i}} \frac{1}{p_{i}}=\frac{1}{2} \frac{w_{\mathrm{f}, j}}{K d p_{k} w_{\mathrm{f}, k}} \frac{1}{p_{i}} \\
& v_{f,(j, k)}^{\prime}=\frac{1}{2} \frac{w_{\mathrm{f}, j}}{N_{\mathrm{f}, k}} \frac{1}{p_{k}}=\frac{1}{2} \frac{w_{\mathrm{f}, j}}{K w_{\mathrm{f}, k}\left[(1-d)+d p_{k}\right]} \frac{1}{p_{k}}(j=i, k) .
\end{aligned}
$$

Equation (B1) also yields simple expressions for the coefficient $r_{j}$ :

$$
\begin{aligned}
& r_{i}=0, \\
& r_{k}=\frac{1-d}{1-d+d p_{k}} .
\end{aligned}
$$

Substituting (B2)-(B4) into expressions for $\Delta W_{i}$ and $\Delta W_{k}$, we get

$$
\Delta W_{i} \propto \frac{1}{p_{i}}-\left(d\left\{\frac{1}{2} \frac{w_{\mathrm{f}, i}}{d p_{k} w_{\mathrm{f}, k}}+\frac{1}{2} \frac{w_{\mathrm{f}, i}}{w_{\mathrm{f}, k}\left[(1-d)+d p_{k}\right]}\right\}+(1-d) \frac{1}{2} \frac{w_{\mathrm{f}, i}}{d p_{k} w_{\mathrm{f}, k}} \frac{1}{p_{i}}\right)
$$

and

$$
\begin{aligned}
\Delta W_{k} \propto \frac{w_{m, k}}{w_{m, i} p_{i}}- & \left\{d\left(\frac{1}{2} \frac{1}{d p_{k}}+\frac{1}{2} \frac{1}{(1-d)+d p_{k}}\right)\right. \\
& \left.+(1-d) \frac{1}{2} \frac{1}{p_{k}\left[(1-d)+d p_{k}\right]}\left(1-\frac{1-d}{1-d+d p_{k}}\right)\right\}
\end{aligned}
$$

The pair $z_{i}=1, z_{k}=0$ are stable whenever $\Delta W_{i}>0$ and $\Delta W_{k}<0$. Simple algebraic rearrangement of these conditions gives 


$$
\frac{w_{\mathrm{m}, i}}{w_{\mathrm{m}, k}}>\frac{p_{k}}{p_{i}} \times\left\{\frac{2\left(1-d p_{i}\right)^{2}}{2 d p_{k}\left[d p_{k}+2(1-d)\right]+(1-d)^{2}}\right\}(i \neq k)
$$

and

$$
\frac{w_{\mathrm{f}, i}}{w_{\mathrm{f}, k}}<\frac{p_{k}}{p_{i}} \times\left[\frac{2 d p_{i}\left(1-d p_{i}\right)}{1-d\left(1-2 d p_{1} p_{2}\right)}\right](i \neq k),
$$

which are general forms of (11), (9) and (12), (10), respectively.

\section{Literature Cited}

Allsop, D. J., and S. A. West. 2004. Sex-ratio evolution in sex changing animals. Evolution 58:1019-1027.

Boomsma, J. J., and A. Grafen. 1991. Colony-level sex ratio selection in the eusocial Hymenoptera. Journal of Evolutionary Biology 4: 383-407.

Brown, G. R., and J. B. Silk. 2002. Reconsidering the null hypothesis: is maternal rank associated with birth sex ratios in primate groups? Proceedings of the National Academy of Sciences of the USA 99: 11252-11255.

Bull, J. J. 1981. Sex ratio evolution when fitness varies. Heredity 46: 9-26.

Cameron, E. Z. 2004. Facultative adjustment of mammalian sex ratios in support of the Trivers-Willard hypothesis: evidence for a mechanism. Proceedings of the Royal Society B: Biological Sciences 271: 1723-1728.

Charnov, E. L. 1979. The genetical evolution of patterns of sexuality: Darwinian fitness. American Naturalist 113:465-480.

. 1982. The theory of sex allocation. Princeton University Press, Princeton, NJ.

Charnov, E. L., R. L. Los-den Hartogh, W. T. Jones, and J. van den Assem. 1981. Sex ratio evolution in a variable environment. Nature 289:27-33.

Clark, A. B. 1978. Sex ratio and local resource competition in a prosimian primate. Science 201:163-165.

Clutton-Brock, T. H., S. D. Albon, and F. E. Guinness. 1982. Competition between female relatives in a matrilocal mammal. Nature 300:178-180.

Cockburn, A., M. P. Scott, and C. R. Dickman. 1985. Sex ratio and intrasexual kin competition in mammals. Oecologia (Berlin) 66: 427-429.

Cockburn, A., S. Legge, and M. C. Double. 2002. Sex ratios in birds and mammals: can the hypotheses be disentangled? Pages 266286 in I. C. W. Hardy, ed. Sex ratios: concepts and research methods. Cambridge University Press, Cambridge.

Courteau, J., and S. Lessard. 2000. Optimal sex ratios in structured populations. Journal of Theoretical Biology 207:159-175.

Day, T., and P. D. Taylor. 2003. Evolutionary dynamics and stability in discrete and continuous games. Evolutionary Ecology Research 5:605-613.

Fisher, R. A. 1930. The genetical theory of natural selection. Clarendon, Oxford.

Frank, S. A. 1987. Individual and population sex allocation patterns. Theoretical Population Biology 31:47-74.

1990. Sex allocation theory for birds and mammals. Annua Review of Ecology and Systematics 21:13-55
. 1998. Foundations of social evolution. Princeton University Press, Princeton, NJ.

Gomendio, M., T. H. Clutton-Brock, S. D. Albon, F. E. Guinness, and M. J. Simpson. 1990. Mammalian sex ratios and variation in costs of rearing sons and daughters. Nature 343:261-263.

Grafen, A. 2006. A theory of Fisher's reproductive value. Journal of Mathematical Biology 53:15-60.

Greenwood, P. J. 1980. Mating systems, philopatry and dispersal in birds and mammals. Animal Behaviour 28:1140-1162.

Greenwood-Lee, J., P. D. Taylor, and D. Haig. 2001. The inclusive fitness dynamics of genomic imprinting. Selection 2:101-116.

Griffin, A. S., B. C. Sheldon, and S. A. West. 2005. Cooperative breeders adjust offspring sex ratios to produce helpful helpers. American Naturalist 166:628-632.

Hamilton, W. D. 1964. The genetical evolution of social behaviour. I, II. Journal of Theoretical Biology 7:1-16, 17-52.

Hardy, I. C. W., ed. 2002. Sex ratios: concepts and research methods. Cambridge University Press, Cambridge.

Herre, E. A. 1985. Sex ratio adjustment in fig wasps. Science 228: 896-898.

Ikawa, T., M. Shimada, H. Matsuda, and H. Okabe. 1993. Sex allocation of parasitic wasps: local mate competition, dispersal before mating and host quality variation. Journal of Evolutionary Biology 6:79-94.

Isaac, J., A. K. Krockenberger, and C. N. Johnson. 2005. Adaptive sex allocation in relation to life-history in the common brushtail possum, Trichosurus vulpecula. Journal of Animal Ecology 74:552558.

Johnson, C. N. 1988. Dispersal and the sex ratio at birth in primates. Nature 332:726-728.

Johnson, C. N., M. Clinchy, A. C. Taylor, C. J. Krebs, P. J. Jarman, A. Payne, and E. G. Ritchie. 2001. Adjustment of offspring sex ratios in relation to availability of resources for philopatric offspring in the common brushtail possum. Proceedings of the Royal Society B: Biological Sciences 268:2001-2006.

Kirkpatrick, M., and R. Lande. 1989. The evolution of maternal characters. Evolution 43:485-503.

Komdeur, J. 1998. Long-term fitness benefits of egg sex modification by the Seychelles warbler. Ecology Letters 1:56-62.

Komdeur, J., S. Daan, J. Tinbergen, and C. Mateman. 1997. Extreme modification of sex ratio of the Seychelles warbler's eggs. Nature 385:522-525.

Kruuk, L. E. B., T. H. Clutton-Brock, S. D. Albon, J. M. Pemberton, and F. E. Guinness. 1999. Population density affects sex ratio variation in red deer. Nature 399:459-461.

Leimar, O. 1996. Life-history analysis of the Trivers and Willard sexratio problem. Behavioral Ecology 7:316-325.

Leturque, H., and F. Rousset. 2003. Joint evolution of sex ratio and 
dispersal: conditions for higher rates of dispersal from good habitats. Evolutionary Ecology 17:67-84.

2004. Intersexual competition as an explanation for sexratio and dispersal biases in polygynous species. Evolution 58: 2398-2408.

Lloyd, P. H., and O. A. E. Rasa. 1989. Status, reproductive success and fitness in Cape mountain zebra (Equus zebra zebra). Behavioral Ecology and Sociobiology 25:411-420.

Palmer, A. R. 2000. Quasireplication and the contract of error: lessons from sex ratios, heritabilities and fluctuating asymmetry. Annual Review of Ecology and Systematics 31:441-480.

Pen, I., and F. J. Weissing. 2000a. Sex ratio optimization with helpers at the nest. Proceedings of the Royal Society B: Biological Sciences 267:539-544.

. 2000b. Sexual selection and the sex ratio: an ESS analysis. Selection 1:59-69.

2002. Optimal sex allocation: steps toward a mechanistic theory. Pages 26-45 in I. C. W. Hardy, ed. Sex ratios: concepts and research methods. Cambridge University Press, Cambridge.

Pen, I., F. J. Weissing, and S. Daan. 1999. Seasonal sex ratio trend in the European kestrel: an ESS analysis. American Naturalist 153: 384-397.

Schino, G. 2004. Birth sex ratio and social rank: consistency and variability within and between primate groups. Behavioral Ecology 15:850-856.

Sheldon, B. C., and S. A. West. 2004. Maternal dominance, maternal condition, and offspring sex ratio in ungulate mammals. American Naturalist 163:40-54.

Shuker, D. M., I. Pen, A. B. Duncan, S. E. Reece, and S. A. West. 2005. Sex ratios under asymmetrical local mate competition: theory and a test with parasitoid wasps. American Naturalist 166: 301-316.

Silk, J. B. 1983. Local resource competition and facultative adjustment of sex ratios in relation to competitive abilities. American Naturalist 121:56-66.

Silk, J. B., E. Willoughby, and G. R. Brown. 2005. Maternal rank and local resource competition do not predict birth sex ratios in wild baboons. Proceedings of the Royal Society B: Biological Sciences 272:859-864.

Taylor, P. D. 1981. Intra-sex and inter-sex sib interactions as sex ratio determinants. Nature 291:64-66.

. 1990. Allele-frequency change in a class-structured population. American Naturalist 135:95-106.

. 1996. Inclusive fitness arguments in genetic models of behaviour. Journal of Mathematical Biology 34:654-674.
Taylor, P. D., and B. J. Crespi. 1994. ESS sex ratios when correlates of relatedness can be assessed. American Naturalist 143:297-316.

Taylor, P. D., and S. A. Frank. 1996. How to make a kin selection model. Journal of Theoretical Biology 180:27-37.

Taylor, P. D., G. Wild, and A. Gardner. 2007. Direct or inclusive fitness: how shall we model kin selection? Journal of Evolutionary Biology 20:296-304.

Trivers, R. L., and H. Hare. 1976. Haplodiploidy and the evolution of the social insects. Science 191:249-263.

Trivers, R. L., and D. E. Willard. 1973. Natural selection of parental ability to vary the sex ratio. Science 179:90-92.

van Schaik, C. P., and S. B. Hrdy. 1991. Intensity of local resource competition shapes the relationship between maternal rank and sex ratios as birth in cercopithecine primates. American Naturalist 138:1555-1562.

Werren, J. H. 1980. Sex ratio adaptations to local mate competition in a parasitic wasp. Science 208:1157-1159.

1984. A model for sex ratio selection in parasitic wasps: local mate competition and host quality effects. Netherlands Journal of Zoology 34:123-143.

Werren, J. H., and G. Simbolotti. 1989. Combined effects of host quality and local mate competition on sex allocation in Larophagus distinguendus. Evolutionary Ecology 3:203-213.

West, S. A., and B. C. Sheldon. 2002. Constraints in the evolution of sex ratio adjustment. Science 295:1685-1688.

West, S. A., S. E. Reece, and B. C. Sheldon. 2002. Sex ratios. Heredity $88: 117-124$.

West, S. A., D. M. Shuker, and B. C. Sheldon. 2005. Sex ratio adjustment when relatives interact: a test of constraints on adaptation. Evolution 59:1211-1228.

Wild, G. 2006. Sex ratios when helpers stay at the nest. Evolution 60:2012-2022.

Wild, G., and P. D. Taylor. 2004. Kin selection models for the coevolution of the sex ratio and sex-specific dispersal. Evolutionary Ecology Research 6:481-502.

. 2005. A kin-selection approach to the resolution of a sexratio conflict between mates. Journal of Theoretical Biology 236: 126-136.

Wild, G., J. Greenwood-Lee, and P. D. Taylor. 2006. Sex allocation and dispersal in a heterogeneous two-patch environment. Theoretical Population Biology 70:225-235.

Yamaguchi, Y. 1985. Sex ratios of an aphid subject to local mate competition with variable maternal fecundity Nature 318:460-462.

Associate Editor: Troy Day Editor: Michael C. Whitlock 